\title{
Fatores de risco na cirurgia das dissecções da aorta ascendente e arco aórtico
}

\author{
Luiz Felipe P. MOREIRA*, Noedir A. G. STOLF*, Caio B. VIANNA*, Paulo M. PÊGO-FERNANDES*, Antônio \\ C. Pereira BARRETO*, Geraldo VERGINELLI*, Adib D. JATENE*
}

RBCCV $44205-32$

\begin{abstract}
MOREIRA, L. F. P.; STOLF, N. A. G.; VIANNA, C. B.; PÊGO-FERNANDES, P. M.; BARRETO, A. C. P.; VERGINELLI, G.; JATENE, A. D. - Fatores de risco na cirurgia das dissecçōes da aorta ascendente e arco aórtico. Rev. Bras. Cir. Cardiovasc., 2 (2): 121-128, 1987.

RESUMO: A experiência cirúrgica no tratamento de 72 pacientes consecutivos com dissecções da aorta proximal foi analisada, com o objetivo de identificar os fatores agravantes do risco da operação. Trinta e nove pacientes foram operados na fase aguda e, em 9 pacientes, o comprometimento era restrito à aorta ascendente. $O$ procedimento cirúrgico mais utilizado foi a substituição da aorta ascendente por tubo de Dacron, associada à correção da delaminação, tendo-se atuado no arco aórtico apenas em 5 pacientes. A mortalidade hospitalar foi de $27,7 \%$, assumindo um valor de $43,5 \%$ para os pacientes operados na fase aguda e de $9 \%$ para os operados cronicamente. Em $45 \%$ desses pacientes houve uma relação direta entre a causa do óbito e a existência de complicaçōes no pré-operatório. Foram considerados como determinantes de maior risco cirúrgico: as lesões neurológicas prévias, o tamponamento cardiaco, o choque cardiogênico, a isquemia miocárdica aguda e a disfunção renal. A compressão do tronco braquiocefálico, ou das artérias carótidas pela dissecção, o orifício de rotura primária da íntima no arco aórtico, a isquemia mesentérica e, nos casos operados na fase aguda, a insuficiência valvar aórtica de moderada ou grave repercussão também foram relacionados a um risco operatório mais elevado. Em conclusão, o resultado do tratamento cirúrgico das dissecçōes da aorta proximal guarda intima relação com as condiçōes pré-operatórias dos pacientes. A obtenção de melhores resultados com a operação na fase aguda depende, principalmente, do reconhecimento precoce da dissecção e da utilização de terapêutica clínica adequada durante o periodo de investigaçāo diagnóstica.
\end{abstract}

DESCRITORES: aorta torácica, cirurgia, fatores de risco.

\section{INTRODUÇÃO}

As dissecçōes aórticas têm alta mortalidade, em nosso meio, principalmente durante a fase aguda. Por outro lado, o reconhecimento dos padrōes clínicos dessa afecção ${ }^{3}{ }^{23}$, assim como o aparecimento de métodos propedêuticos não invasivos ${ }^{13}, 15,27$ têm possibilitado o seu diagnóstico mais precoce, enquanto que avanços importantes também têm sido obtidos no campo de sua terapêutica clínica ${ }^{9} .30 .31$ e cirúrgica ${ }^{4-6}, 8,9,12,17,20,24,28$.
Muitos autores têm referido bons resultados com o tratamento cirúrgico das dissecçōes da aorta ascendente, arco aórtico e aorta descendente na fase crônica ${ }^{1}$. $4 \cdot 9,12,17,20,24,25$. Já a cirurgia realizada na fase aguda ainda representa um desafio, apesar de novas técnicas e propostas ${ }^{2,11,21,26,29}$ e dos melhores resultados obtidos em séries mais recentes ${ }^{6}, 8,9,12,17$. A indicaçāo cirúrgica de urgência é defendida em várias publicaçōes, quando existe o comprometimento agudo da aorta proximal ${ }^{6 \cdot 9}$. 17-20, 22, 23, 30, 31, enquanto que há controvérsias quanto

Trabalho realizado no Instituto do Coração do Hospital das Clínicas da Faculdade de Medicina da Universidade de São Paulo, São Paulo, SP. Brasil.

Apresentado ao 14: Congresso Nacional de Cirurgia Cardiaca, Salvador, BA, 27 e 28 de março, 1987

- Do Instituto do Coraçấo do Hospital das Clinicas da Faculdade de Medicina da Universidade de Sảo Paulo.

Endereço para separatas: Luiz Felipe Moreira. Av. Dr. Enéas Carvalho de Aguiar, 44. Divisāo Cirúrgica. 05403 Sāo Paulo, SP. Brasil. 
MOREIRA, L. F. P.: STOLF. N. A. G.: VIANNA, C. B.: PÉGO-FERNANDES, P. M.: BARRETO. A. C. P.: VERGINELLI, G.: JATENE. A. D. - Fatores de risco na cirurgia das dissecçōes da aorta ascendente e arco aortico. Rev. Bras. Cir. Cardiovasc. $2(2): 121-128,1987$

à indicação do tratamento cirúrgico das dissecções da aorta descendente, nessa fase ${ }^{9}, 19.30 .31$. A influência das condiçōes clinicas pré-operatórias e do grau de comprometimento da aorta sobre os resultados da cirurgia, embora conhecida, tem sido pouco destacada, na literatura ${ }^{7}$ 9. 12, 18. 19 , sendo de grande importância prognóstica, principalmente em pacientes portadores de dissecções agudas ${ }^{8,9,18,19}$.

O propósito desta publicaçāo é analisar as alteraçōes observadas no pré-operatório, a técnica cirúrgica utilizada e os resultados do tratamento cirúrgico das dissecçōes agudas e crônicas da aorta ascendente e arco aórtico proximal, no Instituto do Coração, procurando reconhecer os determinantes de maior risco cirúrgico e apontar as áreas onde deverão se concentrar os esforços futuros para a obtenção de melhores resultados.

\section{MATERIAL E MÉTODOS}

\section{Caracteristicas dos Pacientes}

No período de janeiro de 1983 a dezembro de 1986 , 72 pacientes portadores de dissecçōes da aorta ascendente e arco aórtico proximal foram operados no Instituto do Coração. $O$ tratamento cirúrgico foi realizado na fase aguda, período de até duas semanas após o episódio inicial, em 39 pacientes, e na fase crônica, em 33. Não houve qualquer seleção quanto ao tipo de paciente, só sendo considerada contra-indicação para a cirurgia a existência de morte cerebral documentada.

Sessenta e um pacientes eram do sexo masculino e 11 do feminino. A idade variou de 39 a 72 anos, com média de 56,3 anos, para os pacientes operados na fase aguda, e de 31 a 70 anos, com média de 45,7 anos, para os operados na fase crônica. Quatro pacientes haviam sido anteriormente submetidos a cirurgia de revascularização do miocárdio e outro paciente a correção de aneurisma da aorta abdominal.

O comprometimento da aorta era restrito à aorta ascendente em 9 pacientes, atingindo o arco aórtico e a aorta descendente nos demais. A Figura 1 relaciona a distribuição das dissecções de acordo com a classificação de DeBakey, a fase em que foram operadas e o local de rotura da camada íntima da aorta. O local da rotura da íntima foi estabelecido pelo estudo angiográfico, ou durante a operação.

Em 50 pacientes, a causa mais provável da dissecção foi a hipertensão, estando associada a degeneração mucóide da camada média da aorta. Seis pacientes tinham o diagnóstico de síndrome de Marfan, 3, de comprometimento sifilítico da aorta e 1, de estenose valvar aórtica. Em 2 pacientes, a dissecçāo foi desencadeada por trauma cirúrgico e, em 2 casos, houve lesão da camada intima da aorta, durante a realização de estudo hemodinâmico invasivo. Não se indentificou um fator etiológico, ou predisponente, nos 9 pacientes restantes.

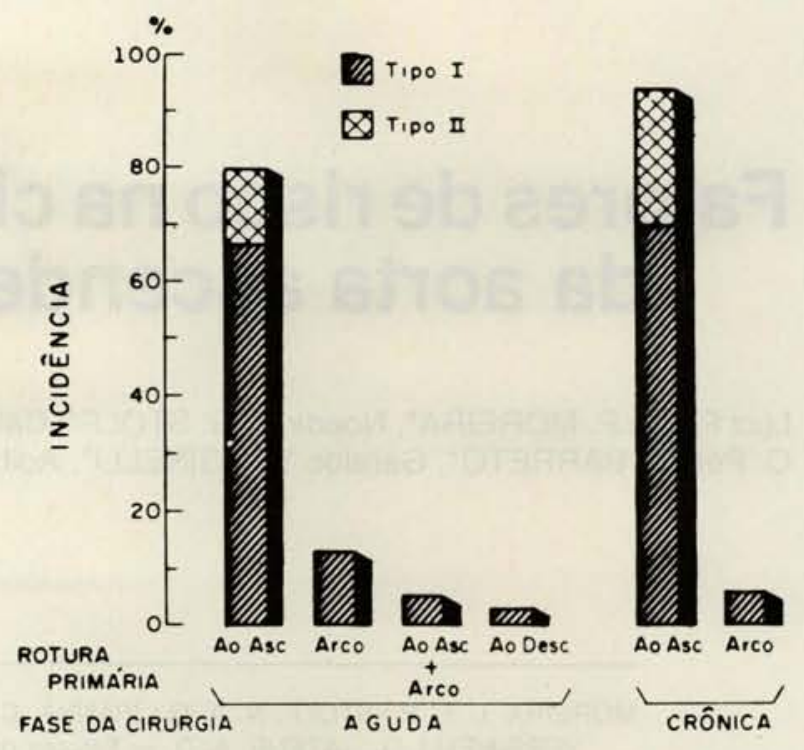

Fig. 1-Correlação entre o tipo de dissecção segundo a classificação de DeBakey, o local de rotura primária da intima e a fase em que foi realizada a cirurgia.

Ao.Asc. $=$ Aorta ascendente $;$ Ao. Desc $=$ Aorta descendente

O intervalo entre o início dos sintomas e a operação variou de duas horas a sete dias, sendo, em média, de 28,3 horas, para os pacientes operados na fase aguda. Esse intervalo variou de três semanas a dois anos, para os pacientes operados na fase crônica. Dor precordial aguda foi o sintoma mais freqüente, sendo referida por 56 pacientes $(77,7 \%)$. Sete pacientes relataram episódios de dor torácica posterior e 2, de dor em membros inferiores, enquanto que dor abdominal foi citada por 13 pacientes, sendo mais freqüente nos casos operados na fase aguda $(28,2 \%)$. Sintomas de insuficiência cardiaca congestiva foram documentados em 28 pacientes e constituíram o quadro clínico predominante, nos pacientes portadores de dissecções crônicas $(75,5 \%)$.

Quarenta e cinco pacientes apresentaram hipertensão ao exame clínico inicial, enquanto que sinais de hipotensāo, ou choque refratário à terapêutica, foram caracterizados em 14 pacientes (19,4\%) e ocorreram, principalmente, em pacientes operados na fase aguda $(33,3 \%)$. As causas dessas alteraçōes foram a existência de tamponamento cardíaco em 8 pacientes e choque cardiogênico, secundário a insuficiência aórtica ou a lesão isquêmica do miocárdio, em 6 pacientes. Hemopericárdio sem repercussão hemodinâmica foi encontrado em 10 pacientes e foram identificadas alteraçōes isquêmicas do miocárdio, ou obstruçōes coronárias secundárias à dissecção, em 9 pacientes, sendo 7 portadores de dissecções agudas.

Diminuição, ou ausência de pulsos em membros superiores ou inferiores, foi documentada em 18 pacientes, sendo que, em 1 caso operado na fase aguda, havia lesão isquêmica grave em membro inferior esquerdo. 
MOREIRA, L. F. P.: STOLF. N. A. G.: VIANNA. C. B.: PÉGO-FERNANDES. P. M.: BARRETO. A. C. P.: VERGINELLI. G.: JATENE. A. D. - Fatores de risco na cirurgia das dissecçōes da aorta ascendente e arco aortico. Rev. Bras. Cir. Cardiovasc. $2(2): 121-128.1987$

A Figura 2 relaciona a ocorrência de disfunção valvar aórtica, de lesão neurológica, de disfunção renal e de isquemia mesentérica, analisando-se separadamente os pacientes portadores de dissecçōes aórticas agudas e crōnicas. A insuficiência aórtica foi avaliada pela angiografia convencional, ou pela ecocardiografia bidimensional. Foram consideradas lesōes neurológicas graves os quadros de coma superficial e a existência de alterações motoras ou sensoriais, enquanto que os quadros de confusão mental foram relacionados como lesões neurológicas leves. Já as alteraçōes da função renal foram caracterizadas pela existência de olingúria e de niveis de creatinina maiores do que $3 \mathrm{mg} / \mathrm{dl} \mathrm{e}$ a isquemia mesentérica foi disgnosticada com base em padrões clínicos e radiológicos, sendo, às vezes, confirmada por exploração cirúrgica.

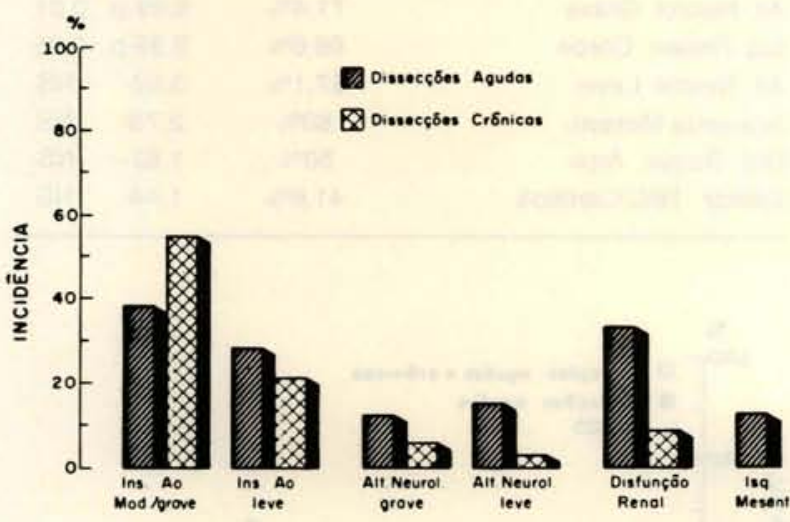

Fig. 2 - Incidência de complicaçōes secundárias à dissecçăo no préoperatório.

O comprometimento do tronco braquiocefálico, ou das artérias carótidas, foi evidenciado angiograficamente em 15 pacientes, enquanto que lesões ateroscleróticas das artérias coronárias foram evidenciadas em 11. Alterações das artérias mesentéricas ou renais foram caracterizadas em 6 pacientes.

\section{Técnica Operatória}

Todos os pacientes foram operados sob circulação extracorpórea clássica e a proteção miocárdica foi obtida com hipotermia tópica e infusāo de solução cardioplégica. Nos casos em que se atuou no arco aórtico, foi realizado breve período de parada circulatória total sob hipotermia profunda.

A substituição da aorta ascendente por tubo de Dacron associada a correção da delaminação, segundo técnica anteriormente descrita ${ }^{23}$, foi realizada em $92,3 \%$ dos pacientes operados na fase aguda e em todos os operados na fase crônica. Em 5 pacientes esse procedimento foi associado a substituição do arco aórtico, ou ao fechamento do orifício primário da dissecção, naquela regiâo. Apenas 3 pacientes portadores de dissecções agudas foram submetidos a aortoplastia.
A suspensão da valva aórtica pela correção da delaminação permitiu a preservação da valva aórtica em $78,8 \%$ dos pacientes com insuficiência daquela valva. Esse procedimento foi realizado em 24 pacientes portadores de dissecções agudas e em 17 pacientes com dissecções crônicas. A substituição da valva aórtica por próteses mecânicas foi realizada em 3 pacientes com alteraçōes degenerativas dos folhetos valvares e, em 8 pacientes portadores de sindrome de Marfan, ou ectasia ânulo-aórtica que foram submetidos a colocação de tubos valvulados com reimplante das artérias coronárias.

Os procedimentos utilizados permitiram a ressecção do local de rotura primária da íntima em 34 pacientes operados na fase aguda $(87,1 \%)$ e em 31 pacientes submetidos a cirurgias na fase crônica $(93,9 \%)$.

Sete pacientes portadores de dissecçōes agudas e 3 com dissecções crônicas também realizaram procedimentos de revascularização do miocárdio, concomitantemente à correção da afecção aórtica.

\section{Análise Estatistica}

Foram considerados, no cálculo da mortalidade operatória, todos os óbitos ocorridos durante o período de hospitalização que se seguiu à época da cirurgia. As influências de 26 caracteristicas pré-operatórias e dados cirúrgicos sobre a mortalidade hospitalar foram analisados, para todos os pacientes do estudo e, separadamente, para os operados na fase aguda. Todas as variáveis foram testadas individualmente, através da análise de contingência pelo teste de Qui-quadrado e os valores médios foram expressos com limites de confiança de $70 \%$.

\section{Resultados}

A mortalidade hospitalar observada neste estudo foi de $27,7 \%$. Dezessete pacientes operados na fase aguda $(43,5 \%)$ faleceram, nesse período, e houve três óbitos cirúrgicos no grupo de pacientes operados na fase crônica $(9 \%)$.

As causas do óbito, estabelecidas em todos os pacientes através de necrópsia, estão na Tabela 1. Em 9 pacientes $(45 \%)$, houve uma relação direta entre a causa do óbito e a existência de disfunções pré-operatórias em órgãos vitais. Seis pacientes $(30 \%)$ apresentaram complicações pós-operatórias diretamente relacionadas a alteraçōes anteriores à cirurgia, que podem ter contribuído para o óbito e, em apenas 5 casos (25\%), a causa do óbito não pôde ser relacionada a fatores agravantes do risco cirúrgico. 
MOREIRA, L. F. P.: STOLF, N. A. G.: VIANNA. C. B.: PÉGO-FERNANDES. P. M.: BARRETO. A. C. P.: VERGINELLI. G : JATENE. A. D. - Fatores de risco na cirurgia das dissecçōes da aorta ascendente e arco aortico. Rev. Bras Cir. Cardiovasc. $2(2): 121-128,1987$

TABELA 1

CAUSAS DA MORTALIDADE HOSPITALAR

\begin{tabular}{ll}
\hline & (n) $(\%)$ \\
\hline Sindrome de Baixo Débito & $5-25 \%$ \\
Sangramento Rotura da aorta & $2-10 \%$ \\
Redissecção Aguda & $1-5 \%$ \\
Compl. Neurológica & $1-5 \%$ \\
Compl. Pulmonar & $4-20 \%$ \\
Insuficiência Renal & $2-10 \%$ \\
Infarto Mesentérico & $1-5 \%$ \\
Septicemia & $4-20 \%$ \\
\hline
\end{tabular}

A Tabela 2 mostra a incidência de complicações no periodo pós-operatório, excetuando-se os pacientes que apresentaram óbito na operação. Em $26 \%$ dos casos, essas complicaçōes já existiam no periodo pré-operatório.

TABELA 2

COMPLICACÓES PÓS-OPERATÓRIAS

\begin{tabular}{lc}
\hline & (n) $(\%)$ \\
\hline Síndrome de Baixo Débito & $9-13,2 \%$ \\
Reoperação por Sangramento & $4-5,9 \%$ \\
Compl. Neurológica Grave & $15-22 \%$ \\
Compl. Neurológica Leve & $11-16,1 \%$ \\
Compl. Pulmonar & $19-27.9 \%$ \\
Insuficiência Renal & $15-22 \%$ \\
\hline
\end{tabular}

Os fatores pré-operatórios e os dados cirúrgicos que foram considerados como agravantes do risco cirúrgico, analisando-se, conjuntamente, os pacientes operados nas fases aguda e crōnica, estão relacionados na Tabela 3. A influência da idade sobre o resultado da cirurgia, nesses pacientes, foi analisada na Figura 3. Não houve qualquer relação da mortalidade hospitalar com o sexo dos pacientes, sintomas pré-operatórios, etiologia da dissecção, cirurgias cardiacas prévias, existência ou grau de insuficiência valvar aórtica, alterações de pulsos periféricos, perfusāo de órgāos abdominais, ou de membros pela falsa luz, lesões ateroscleróticas das artérias coronárias, achado cirúrgico de hemopericárdio, técnica cirúrgica utilizada, ou associação a outros procedimentos cirúrgicos.

A Tabela 4 relaciona os fatores de risco cirúrgico, quando se analisam apenas os pacientes operados na fase aguda. Não houve qualquer influência da idade dos pacientes sobre os resultados observados nesse grupo (Figura 3), assim como dos fatores que também não foram considerados como agravantes do risco cirúrgico, no grupo total de pacientes estudados, à exceção da insuficiência aórtica, de moderada ou grave repercussão hemodinâmica.

TABELA 3

DETERMINANTES DO RISCO CIRÜRGICO EM PACIENTES PORTADORES DE DISSECCÓES AGUDAS E CRÓNICAS DA AORTA PROXIMAL

\begin{tabular}{lcccc}
\hline \multicolumn{1}{c}{ FATORES } & MORT & \multicolumn{2}{c}{ QUI. } \\
DE RISCO & HOSP & QUADRADO \\
\hline Cirurgia Emerg. & $43,5 \%$ & $10,58-\mathrm{p}$ & 0,005 \\
Tampon. Cardiaco & $75 \%$ & $10,17-\mathrm{p}$ & 0,005 \\
Disf. Renal & $56,2 \%$ & $8,06-\mathrm{p}$ & 0,005 \\
Choque Cardiog. & $83,3 \%$ & $6,99-\mathrm{p}$ & 0.01 \\
Alt. Neurol. Grave & $71,4 \%$ & $6,99-\mathrm{p}$ & 0,01 \\
Isq. Dissec. Coron. & $66,6 \%$ & $3,95-\mathrm{p}$ & 0,05 \\
Alt. Neurol. Leve & $57,1 \%$ & $3,52-$ & $\mathrm{NS}$ \\
Isquemia Mesent. & $60 \%$ & $2,73-$ & $\mathrm{NS}$ \\
Orif. Dissec. Arco & $50 \%$ & $1,82-$ & $\mathrm{NS}$ \\
Compr. TBC/Carótida & $41,6 \%$ & $1,44-$ & $\mathrm{NS}$ \\
\hline
\end{tabular}

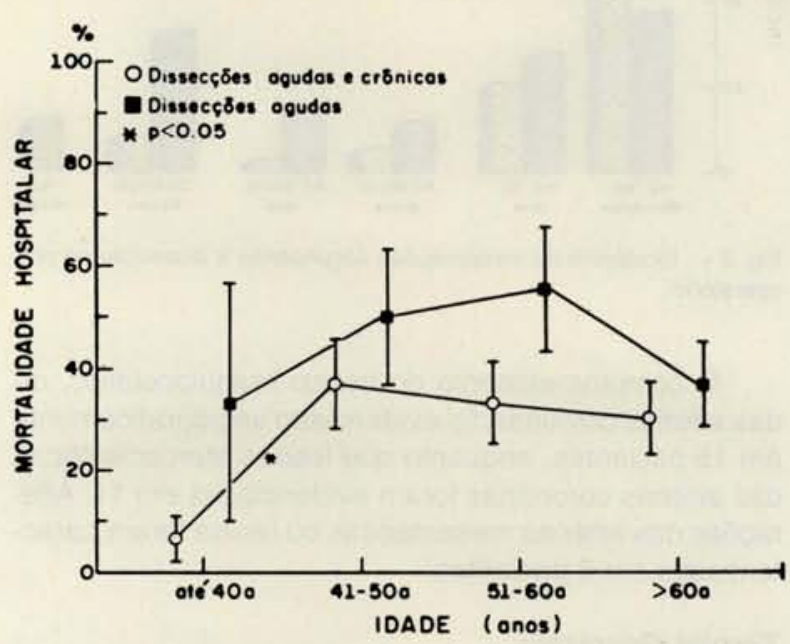

Fig. 3-Correlação entre a idade dos pacientes operados e a mortalidade hospitalar.

A separação dos pacientes do estudo em quatro grupos, de acordo com as características pré-operatórias e os dados operatórios considerados como determinantes de maior risco cirúrgico, foi realizada com o objetivo de analisar a influência da associação de múltiplos fatores sobre os resultados do tratamento cirúrgico, em todos os pacientes e, separadamente, nos operados na fase aguda. Forar considerados como fatores maiores: as lesões neuroıogicas graves, os quadros de tamponamento ou choque cardiogênico, a isquemia miocárdica ou as obstruções coronárias secundárias à dissecção e as alterações da função renal. Já as lesões neurológicas leves, a isquemia mesentérica, a compressão do 
MOREIRA, L. F. P.: STOLF. N. A. G.: VIANNA. C. B.: PEGO-FERNANDES. P. M.. BARRETO. A. C. P.: VERGINELLI. G.: JATENE. A. D. - Fatores de risco na cirurgia das dissecçōes da aorta ascendente e arco aortico. Rev Bras. Cir. CardiovasC. $2(2): 121-128.1987$

tronco braquiocefálico ou das artérias carótidas pela dissecção e a existência do orifício primário da dissecção no arco aórtico foram considerados como fatores menores. A insuficiência aórtica de moderada ou grave repercussāo só foi incluida como fator menor quando analisados separadamente os casos operados na fase aguda. A Figura 4 relaciona a mortalidade hospitalar para os quatro grupos de risco, considerando-se, conjuntamente, todos os pacientes e, separadamente, os portadores de dissecçōes agudas. O Grupo I se refere aos pacientes que apresentaram no máximo um fator menor. Já o Grupo Il é caracterizado pela presença de pelo menos um fator maior, ou dois menores, enquanto que o Grupo III se refere aos pacientes que apresentaram um fator maior associado a outro fator maior, ou a dois menores. O Grupo IV reúne os pacientes com, no minimo, dois fatores maiores associados a dois menores, ou a outro fator maior.

TABELA 4

DETERMINANTES DO RISCO CIRÚRGICO EM PACIENTES PORTADORES DE DISSECCÖES AGUDAS DA AORTA PROXIMAL

\begin{tabular}{lcccc}
\hline $\begin{array}{l}\text { FATORES } \\
\text { DE RISCO }\end{array}$ & $\begin{array}{c}\text { MORT. } \\
\text { HOSP. }\end{array}$ & \multicolumn{2}{c}{$\begin{array}{c}\text { QUI- } \\
\text { QUADRADO }\end{array}$} \\
\hline Alt. Neurol. Grave & $100 \%$ & $7,3-\mathrm{p}$ & 0,01 \\
Choque Cardiog. & $83,3 \%$ & $4,61-\mathrm{p}$ & 0,05 \\
Tampon. Cardiaco & $75 \%$ & $3,99-\mathrm{p}$ & 0,05 \\
Isq. Dissec. Coron. & $71,4 \%$ & $2,84-$ & $\mathrm{NS}$ \\
Disf. Renal & $61,5 \%$ & $2,48-$ & $\mathrm{NS}$ \\
Alt. Neurol. Leve & $66,6 \%$ & $1,57-$ & $\mathrm{NS}$ \\
Insuf. Aórtica Imp. & $53,3 \%$ & $0,99-$ & $\mathrm{NS}$ \\
Isquemia Mesent. & $60 \%$ & $0,59-$ & $\mathrm{NS}$ \\
Compr. TBC/Carótida & $57,1 \%$ & $0,57-$ & $\mathrm{NS}$ \\
Orif. Dissec. Arco & $57,1 \%$ & $0,57-$ & $\mathrm{NS}$ \\
\hline
\end{tabular}

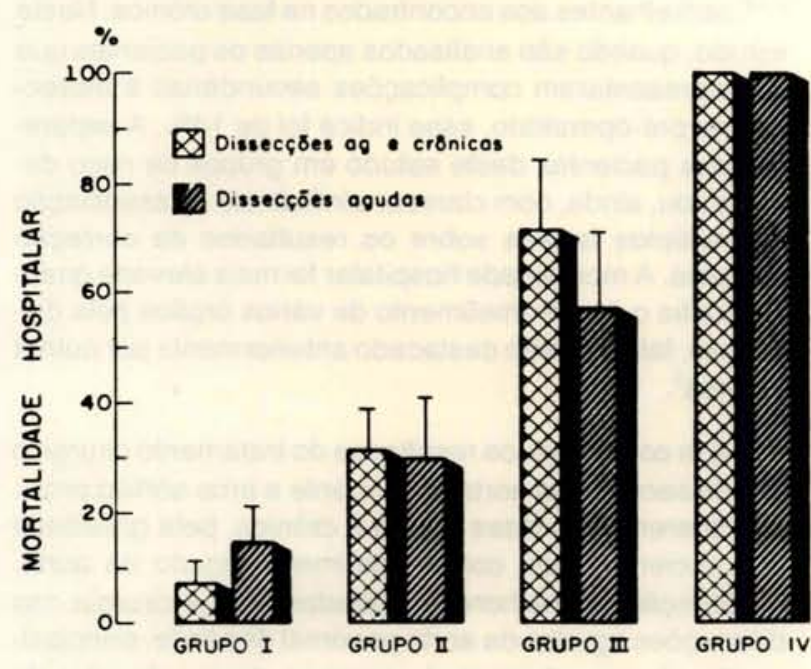

Fig. 4-Divisão dos pacientes em grupos de risco, segundo a ocorrência de múltiplos fatores agravantes do resultado da cirurgia.

\section{DISCUSSĀO}

Muitos autores têm discutido os aspectos diagnósticos, a conduta terapêutica e os resultados de diferentes técnicas operatórias no tratamento de pacientes portadores de dissecçōes da aorta torácica. É destacada a importância do quadro clinico ${ }^{3.23}$ e dos métodos propedêuticos não invasivos ${ }^{13.15 .27}$ para o diagnóstico precoce dessa afeç̧ão. Em relação à terapêutica, aponta-se com uniformidade o tratamento cirúrgico como a conduta de escolha para as dissecçōes aórticas na fase crônica'. 4.9. 12. 17, 20. 24. 25, enquanto que essa posição é defendida apenas para as dissecçôes da aorta próximal na fase aguda $^{6-9}$. 17. 20. 22. 23. 30. 31. São relatados resultados semeInantes com o tratamento clinico e com a cirurgia no controle das dissecçōes agudas da aorta descendente ${ }^{9}$. 19. 30. 31 .

A correção cirúrgica das dissecçōes crônicas da aorta ascendente apresenta indices de mortalidade hospitalar que variam entre 4 e $20 \%{ }^{1.6 .}$. 8. 9. 17.20 . 25 , sendo relatada uma sobrevida média de $75 \%$ dos pacientes operados, ao final de cinco anos ${ }^{1.8 .}{ }^{8} 12$ e de $56 \%$ aos 10 anos de seguimento ${ }^{12}$. São referidos indices de mortalidade mais elevados, variando de 20 a $50 \%$, na abordagem cirúrgica do arco aórtico ${ }^{5.6},{ }^{10,24}$, apesar da utilização das técnicas de hipotermia profunda ${ }^{5}$, ou de perfusão seletiva de ramos da aorta $^{10}$. Resultados semelhantes foram observados no tratamento das dissecçōes crônicas da aorta proximal, neste estudo, tendo-se realizado a ressecçāo do local de rotura primária da camada íntima da aorta em $93 \%$ desses pacientes. Destaca-se, na literatura, que a permanência do orifício de entrada das dissecções não tem influenciado os resultados imediatos do tratamento cirúrgico ${ }^{18}$, embora seja um fator de risco importante na evolução tardia dos pacientes ${ }^{12}$.

Os melhores resultados da terapêtica cirúrgica no tratamento das dissecções crônicas da aorta proximal devem-se, principalmente, à baixa incidência de complicaçōes secundárias à dissecção, nessa fase. $A$ insuficiência aórtica, que é observada com freqüência na fase crônica, não tem elevado o risco cirúrgico, nesses pacientes, independentemente da técnica empregada para a sua correção ${ }^{8}$. 9. 18, 19. A substituição da valva aórtica tem sido mais utilizada no tratamento da insuficiência aórtica secundária às dissecçōes da aorta ascendente ${ }^{8}$. 9. 22,25 do que os procedimentos conservadores ${ }^{9}$. 17. 28 . Já o emprego de tubos valvulados tem sido descrito, principalmente, nas ectasias ânulo-aórticas, ou na síndrome de Marfan 1, 4. 6. ${ }^{16}$. Neste grupo de pacientes, foi possivel conservar a valva aórtica em $62,9 \%$ dos casos com comprometimento valvar na fase crônica e em $96 \%$ dos pacientes com dissecções agudas e insuficiência aórtica.

Em relação às operações realizadas na fase aguda, os índices de mortalidade imediata diferem muito, mesmo quando comparamos os resultados dos grupos de maior experiência ${ }^{6} .8,9,{ }^{17}$. Nas dissecçōes da aorta ascendente e do arco aórtico, esses índices variam de 
MOREIRA, L. F. P.: STOLF. N. A G.: VIANNA, C. B.: PEGO-FERNANDES. P. M.: BARRETO, A. C. P : VERGINELLI. G : JATENE. A. D. - Fatores de risco na cirurgia das dissecçoes da aorta ascendente e arco aortico. Rev. Bras Cir Cardiovasc. $2(2): 121-128,1987$

7 a $47 \%$ 6. 9, 17, 20, 22, 28 . Por outro lado, levanta-se a possibilidade desses estudos analisarem grupos diferentes de pacientes. Os casos que apresentam comprometimento de múltiplos órgãos, ou alteraçōes importantes da funçāo circulatória, ou neurológica, pela dissecção, têm sido considerados como contra-indicação à operação na fase aguda, por alguns autores ${ }^{9}{ }^{18},{ }^{31}$. Deve-se destacar, ainda, que existem Serviços que recebem pacientes em caráter de emergência, com uma freqüência maior, fato que pode influenciar o tipo de pacientes submetidos à terapêutica cirúrgica, por esses grupos.

Novas técnicas têm sido propostas, para a correção das dissecções aórticas na fase aguda, embora sejam responsáveis por índices de mortalidade semelhantes aos relatados anteriormente $2.11,14,21,26$. O uso de próteses intraluminares e da cola biológica tem permitido a diminuição do tempo de parada anóxica e do sangramento após a operaçāo ${ }^{11}$ 14. 21, enquanto outras técnicas visam prevenir a redissecção ${ }^{2}{ }^{26}$. São relatados indices de redissecção, que variam de 13 a $25 \%$ com a substituição da aorta por enxerto tubular, associada à correção da delaminação distal ${ }^{\beta, 12}$.

Por outro lado, estudos recentes têm mostrado resultados promissores na correção das dissecções aórticas agudas $^{6,8,17,21}$ e apontam uma nítida relaçāo entre as complicaçōes decorrentes do comprometimento da aorta no pré-operatório e as conseqüências da operação ${ }^{8,18}$. Neste trabalho, foi possivel notar uma correlação direta entre as causas do óbito e alterações pré-operatórias causadas pela dissecção, em $45 \%$ dos pacientes analisados.

A determinação dos fatores agravantes do risco cirúrgico demonstrou que a existência de alterações neurológicas graves no pré-operatório foi responsável por uma mortalidade elevada, neste estudo, existindo controvérsias, na literatura, em relaçāo à oportunidade do tratamento cirúrgico, nessa situação. Alguns autores defendem a tentativa de reverter as complicaçōes neurológicas antes da realização da operação da aorta na fase aguda $^{29}$, enquanto outros encaram essas complicações como uma contra-indicaçāo à correçāo cirúrgica da dissecção, naquela fase ${ }^{9.18}$

O tamponamento cardiaco, o choque cardiogênico e a isquemia miocárdica também foram considerados determinantes de maior risco cirúrgico, nestes pacientes, à semelhança de outros relatos da literatura ${ }^{9}{ }^{18},{ }^{19}$. Do mesmo modo, as disfunções renais influenciaram, diretamente, a mortalidade nas dissecçōes da aorta proximal neste trabalho, sendo referida por outros autores uma relação importante entre as alteraçōes da perfusão renal, ou de vasos abdominais, e os resultados da cirurgia na fase aguda ${ }^{19}$. Por outro lado, não foi significativa a influência da isquemia mesentérica sobre a mortalidade hospitalar, neste estudo, ao contrário de outros relatos da literatura ${ }^{9,19}$
Quando se analisa a idade dos pacientes operados, destaca-se apenas a existência de melhores resultados no grupo de pacientes com menos de 40 anos. Isto pode ser explicado pelo fato de que os pacientes mais jovens foram submetidos ao tratamento cirúrgico predominantemente na fase crônica, como já destacado anteriormente, por outros autores ${ }^{8.18}$

O orifício da dissecção no arco aórtico também têm sido considerado um fator de risco na correção das dissecçōes aórticas ${ }^{18}$, embora nāo tenha sido correlacionado significativamente com os índices de mortalidade dos pacientes aqui analisados. Fato semelhante foi observado com a compressão do tronco braquiocefálico, ou das artérias carótidas, pela dissecção, alterações também citadas como agravantes do risco cirúrgico no tratamento das dissecções da aorta proximal ${ }^{9}$.

Já a existência de insuficiência aórtica de moderada ou grave repercussão hemodinâmica chegou a influenciar apenas a mortalidade dos pacientes operados na fase aguda, neste estudo, mas sem significância estatistica. Outros grupos destacam que a presença de insuficiência aórtica não tem alterado os resultados do tratamento das dissecçōes aórticas proximais ${ }^{9}{ }^{18}{ }^{19}$. por ser possivel a correçāo completa dessa alteração com a operação $^{18}$.

Enquanto as complicaçōes mais importantes das dissecções aórticas interferem, efetivamente, nos resultados da terapêutica cirúrgica, nāo se verificou, neste trabalho, e também nāo tem sido referida na literatura, qualquer relaçāo do risco cirúrgico com a etiologia da dissecção, o quadro clínico inicial, ou as técnicas operatórias empregadas ${ }^{8,9,18,19}$

Finalmente, a realização da operação em caráter de urgência, associada a menor incidência de fatores de risco, tem permitido a obtenção de índices de mortalidade mais baixos no tratamento das dissecçōes agudas ${ }^{8}$. 9.18 , semelhantes aos encontrados na fase crōnica. Neste estudo, quando são analisados apenas os pacientes que não apresentaram complicaçōes secundárias à dissecção no pré-operatório, esse indice foi de $14 \%$. A separação dos pacientes deste estudo em grupos de risco demonstrou, ainda, com clareza, a influência da associação de múltiplos fatores sobre os resultados da correção cirúrgica. A mortalidade hospitalar foi mais elevada quando existia o comprometimento de vários órgãos pela dissecção, fato também destacado anteriormente por outros autores $^{9}$.

Em conclusão, os resultados do tratamento cirúrgico das dissecçōes da aorta ascendente e arco aórtico proximal diferem, nas fases aguda e crônica, pela gravidade dos pacientes com comprometimento agudo da aorta. A obtenção de melhores resultados com a cirurgia nas dissecções agudas da aorta proximal depende, principalmente, do reconhecimento precoce dessa afecção, do controle clínico adequado durante o período de investi- 
MOREIRA. L. F. P : STOLF. N. A. G.: VIANNA, C. B : PĖGO-FERNANDES. P. M.: BARRETO. A. C. P.: VERGINELLI. G.: JATENE. A. D. - Fatores de risco na cirurgia das dissecçōes da aorta ascendente e arco aortico. Rev Bras Cir. Cardiovasc $2(2): 121-128,1987$

gação diagnóstica e da indicação da cirurgia em caráter de urgência. A existência de complicaçōes secundárias à dissecção, no pré-operatório, eleva, significativamente, o risco da operação. Finalmente, o comprometimento de múltiplos órgãos pela dissecção pode ser considerado uma contra-indicaçāo à terapêutica cirúrgica.

RBCCV 44205-32

MOREIRA, L. F. P.; STOLF, N. A. G.; VIANNA, C. B.; PEGO-FERNANDES, P. M.; BARRETO, A. C. P.: VERGINELLI, G.; JATENE, A. D. - Risk factors in surgery for thoracic aortic dissection. Rev. Bras. Cir. Cardiovasc., 2 (2): 121-128, 1987

ABSTRACT: The surgical experience in the treatment of 72 patients with proximal aortic dissections was analized to identify the determinants of high operative risk. Thirty-seven patients were operated upon in the acute stage and the dissection was restrict to ascending aorta in 9. The most common surgical procedure was the interposition of Dacron graft in the ascending aorta associated to obliteration of false lumen. The aortic arch was approached only in 5 patients. The overall operative mortality was $27.7 \%$ and patients with acute dissections had mortality rate of $43.5 \%$. There was a direct relationship between the causes of death and preoperative complications in $45 \%$ of these cases. The following factors were considered predictors of high operative risk: previous neurologic compromise, cardiac tamponade, persistent shock, acute myocardial ischemia and renal dysfunction. Involvement of innominate or carotid arteries by dissection, presence of intimal tear in aortic arch, mesenteric ischemia and only in the acute cases, moderate or severe aortic valve incompetence were also correlated with a higher operative mortality. In conclusion, the operative risk of the treatment of proximal aortic dissection has a relationship with preoperative clinical condition. Better results in the acute stage could be improved by early diagnosis and adequate medical therapy during investigation period.

DESCRIPTORS: thoracic aorta, surgery, risk factors.

\section{REFERÊNCIAS BIBLIOGRÁFICAS}

1 CABROL, C.; PAVIE, A.; MESNILDREY, P.; GRANDJBAKHCH, I.; LAUGHLIN, L.; BORS, V.; CORCOS, T. - Long-term results with total replacement of the ascending aorta and reimplantation of the coronary arteries. J. Thorac. Cardiovasc. Surg., 91 (1): 17-25, 1986.

2 CARPENTIER, A.; DELOCHE, A.; FABIANI, J. N.; CHAUVAUD, S.; RELLAND, J.; NOTTIN, R.; VOUCHE, P.; MASSOUD, H.; DUBOST, C. - New surgical approach to aortic dissection: flow reversal and thromboexclusion. J. Thorac. Cardiovasc. Surg., 81 (5): 659-668, 1981.

3 COOKE, J. P. \& SAFFORD, R. E. - Progress in the diagnosis and management of aortic dissections. Mayo Clin. Proc., 61 (2): 147-153, 1986.

4 CRAWFORD, E. S.; CRAWFORD, J. L.; STOWE, C. L. SAFI, H. J. - Total aortic replacement for chronic aortic dissection occuring in patients with or without Marfan's syndrome. Ann. Surg., 199 (3): 358-362, 1984.

5 CRAWFORD, E. S. \& SNYDER, D. M. - Treatment of aneurysms of the aortic arch: a progress report. J. Thorac. Cardiovasc. Surg., 85 (2): 237-246, 1983.

6 CULLIFORD, A. T.; AYVALIOTIS, B.; SHEMIN, R.; COLVIN, S. B.; ISOM, O. W.; SPENCER, F. C. - Aneurysms of the ascending aorta and transverse arch: surgical experience in 80 patients. J. Thorac. Cardiovasc. Surg., 83 (5): 701-710, 1982.
7 DeBAKEY, M. E.; HENLY, W. A.; COOLEY, D. A.; MORRIS, G. C.; CRAWFORD, E. S.; BEALL, A. C. - Surgical management of dissecting aneurysms of the aorta J. Thorac. Cardiovasc. Surg., 49 (1): 130-149, 1965.

8 DeBAKEY, M. E.; McCOLLUM, C. H.; CRAWFORD, E. S.; MORRIS, G. C.; HOWELL, J.; NOON, G. P.; LAWRIE, G. - Dissection and dissecting aneurysms of the aorta: twenty-year follow-up of five hundred twentyseven patients treated surgically. Surgery, $92(6)$ : 1118-1134, 1982.

9 DOROGHAZI, R. M.; SLATER, E. E.; DeSANCTIS, R. W.; BUCKLEY, M. J.; AUSTEN, W. G.; ROSENTHAL, S. - Long-term survival of patients with treated aortic dissection. J. Am. Coll. Cardiol., 3 (4): 1026-1034, 1984.

10 FRIST, W. H.; BALDWIN, J. C.; STARNES, V. A.; STINSON, E. B.; OYER, P. E.; MILLER, D. G.; JAMIESON. S. W.; MITCHELL, R. S.; SHUMWAY, N. E. - A reconsideration of cerebral perfusion in aortic arch replacement. Ann. Thorac. Surg., 42 (3): 273-281, 1986.

11 GUILMET, D.; BACHET, J.; GOUDOT, B.; LAURIAN, C.; GIGOU, F.; BICAL, O.; BARBAGELATTA, M. - Use of biological glue in acute aortic dissection: preliminary clinical results with a new surgical technique. J. Thorac. Cardiovasc. Surg., 77 (4): 516-521, 1979.

12 HAVERICH, A.; MILLER, D. G.; SCOTT, W. C.; MITCHELL, R. S.; OYER, P. E.; STINSON, E. B.; SHUMWAY, N. E. - Acute and chronic aortic dissections: determinants of long-term outcome for operative sirvivors. Circulation, 72 (Supl. 3): 22-34, 1985. 
MOREIRA, L. F. P.: STOLF. N. A. G.: VIANNA, C. B.: PËGO-FERNANDES. P. M.: BARRETO. A. C. P.: VERGINELLI. G : JATENE.

A. D. - Fatores de risco na cirurgia das dissecçōes da aorta ascendente e arco aortico. Rev. Bras Cir Cardiovasc.

$2(2): 121-128,1987$

HILL, J. A.; LAMBERT, C. R.; AKINS, E. W.: CARMICHAEL, M. J. - Ascending aortic dissections: detection by M. R. I. Am. Heart J., 110 (4): 894-896, 1985.

KOYAMADA, K.; ISHIKAWA, S.; YAMAKI, S.; KAKIHATA, H. - Surgical treatment for dissecting aneurysm of the aorta using double ringed grafts. J. Cardiovasc. Surg. (Torino), 26 (5): 488-493, 1985.

15 MATHEW, T. \& NANDA, N. C. - Two-dimensional and doppler echocardiografic evaluation of aortic aneurysms and dissections. Am. J. Cardiol., 54 (3): 379-385, 1984.

16 McDONALD, G. R.; SCHAFF, H. V.; PYERITZ, R. E.; MCKUSICK, V. A.; GOTT, V. L. - Surgical management of patients with the Marfan's syndrome and dilatation of the ascending aorta. J. Thorac. Cardiovasc. Surg., 81 (2): 180-186, 1981.

17 MILLER, D. C.; STINSON, E. B.; OYER, P. E.; ROSSITER, S. J.; REITZ, B. A.; GRIEPP, R. B.; SHUMWAY, N. E. - Operative treatment of aortic dissections: experirience with 125 patients over a sixteen-year period. J. Thorac. Cardiovasc. Surg., 78 (3): 365-382, 1979.

18 MILLER, D. G.; MITCHELL, R. S.; OYER, P. E.; STINSON, E. B.; JAMIESON, S. W.; SHUMWAY, N. E. - Independent determinants of operative mortality for patients with aortic dissections. Circulation, 70 (Supl. 1): $153-164,1984$

19 PINET, F.; FROMENT, J. C.; GUILLOT, M.; GOURDOT, Y.; MEYER, P.; LOIRE, R.; TOUBOUL, P.; DELAHAYE, J. P.; BIRON, A.; MESSY, P. - Prognostic factors and indications for surgical treatment of acute aortic dissections: a report based on 191 observations. Cardiovasc. Intervent. Radiol., 7 (2): 257-266, 1984.

PRESSLER, V. \& MCNAMARA, J. J. - Aneurysm of thorac aorta: review of 260 cases. J. Thorac. Cardiovasc. Surg., 89 (1): 50-54, 1985.

21 RIZZOLI, G.; ARU, G.; MILANO, A.; VALFRE, C.; MAZZUCCO, A.; RUBINO, M.; GALLUCCI, V. - The use of intraluminal prosthesis in the surgical management of aortic dissections. Thorac. Cardiovasc. Surg., 34 (2): 88-91, 1986.
22 SAMPAIO, F. A. F.: VILA, J. H.: MELO, R. F. A : BITTENCOURT, D.: SOUZA, M. C.; GALIANO, N.: MACRUZ. R.; ZERBINI, E. J. - Dissecção aguda da aorta: seguimento imediato e tardio de pacientes submetidos a tratamento clinico e cirúrgico. Arq. Bras. Cardiol., 45 (6): $385-387,1985$.

23 StOlF. N. A. G. - Dissecçōes aórticas. Rev. Bras. Med. 40 (9): $331-337,1983$

24 STOLF, N. A. G.; FERNANDES, P. M. P.: BITTENCOURT D.: BARRETO, A. C. P.: VERGINELLI, G.; PILEGGI, F.; ZERBINI, E. J. - Aneurismas e dissecçōes da aorta toracica: tratamento cirúrgico. Arq. Bras. Cardiol. 41 (4): 221-226, 1983.

25 STOLF, N. A. G.; FERNANDES, P. M. P.: COSTA, R. BARRETO, A. C. P.; AMARAL, R. V. G.; PILEGGI, F.; VERGINELLI, G. - Tratamento cirúrgico das dissecçōes crônicas da aorta torácica. Rev. Hosp. Clin. Fac. Med. S. Paulo, 38 (1): 3-7, 1983.

26 TANABE, T.; HASHIMOTO, M.; SAKAI, K.; YASUDA, K.; TAKEOKA, T.; MATSUNANI, O ; SAKUMA, M.; GOHDA, T. - Surgical treatment of aortic dissection: aplication of ivalon sponge to dissected lumen. Ann. Thorac. Surg., 41 (2): 169-175, 1986.

27 THORSEN, M. K.; SAN DRETTO, M. A.; LAWSON, T. L.; FOLEY, W. D.; SMITH, D. F; BERLAND, L. L. Dissecting aortic aneurysms: accuracy of computed tomografic diagnosis. Radiology, 148 (3): 773-777, 1983.

28 VILJANEN, T.; LANDTMAN, M.; LUOSTO, R. - Late results of the surgical treatment of aortic dissections. Thorac. Cardiovasc. Surg., 33 (1): 8-15, 1985.

29 WALteRBUSCH, G.; OELERT, H.; BORST, H. G. - Restoration of cerebral blood flow by extra anatomic bypass in acute aortic dissection. Thorac. Cardiovasc. Surg., 32 (6): $381-382,1984$

30 WHEAT Jr., M. W.: HARRIS, P. D.; MALM, M. R.; KAISER, G.; BOWMAN Jr., F. O.; PALMER, R. F. - Acute dissecting aneurysms of the aorta. J. Thorac. Cardiovasc. Surg., 58 (3): 344-381, 1969.

31. WOLFE, W. G. \& MORAN, J.F. - The evolution of medical and surgical management of acute aortic dissection. Circulation, 56 (4): 503-505, 1977. 